\title{
A LOWER ESTIMATE FOR THE NORM OF THE KERZMAN-STEIN OPERATOR
}

\author{
MICHAEL BOLT
}

\begin{abstract}
We establish an elementary lower estimate for the norm of the Kerzman-Stein operator for a smooth, bounded domain. The estimate involves the boundary length and logarithmic capacity. The estimate is tested on model domains for which the norm is known explicitly. It is shown that the estimate is sharp for an annulus and a strip, and is asymptotically sharp for an ellipse and a wedge.
\end{abstract}

1. Introduction. Suppose $\Omega \subset \subset \mathbf{C}$ is a continuously differentiable, multiply connected domain in the plane and $L^{2}(\partial \Omega)$ is the space of square-integrable functions defined with respect to arclength measure on the boundary. The Cauchy singular operator on $L^{2}(\partial \Omega)$ can be expressed using a principal value integral,

$$
\mathcal{C}_{0} f(z)=\frac{1}{2 \pi i} \mathrm{P} . \mathrm{V} \cdot \int_{\partial \Omega} \frac{f(w) d w}{w-z} \text { for } \quad z \in \partial \Omega .
$$

It is known classically that $\mathcal{C}_{0}$ is bounded on $L^{2}(\partial \Omega)$, so its skewhermitian part, $\mathcal{A}=\mathcal{C}_{0}-\mathcal{C}_{0}^{*}$, is also bounded. In fact, Lanzani $[\mathbf{8}]$ showed that with these conditions $\mathcal{A}$ is compact-it acts by integration against the kernel,

$$
A(z, w)=\frac{1}{2 \pi i}\left[\frac{T(w)}{w-z}-\frac{\overline{T(z)}}{\bar{w}-\bar{z}}\right] \text { for } w, z \in \partial \Omega,
$$

where $T(w)$ is the positively-oriented unit tangent vector at $w \in \partial \Omega$. (The apparent singularities cancel each other.) The operator $\mathcal{A}$ is known as the Kerzman-Stein operator for $\Omega$. Kerzman and Stein used this operator to give an explicit construction for the Szegö kernel and, in so doing, they found an elegant way to compute the Riemann map [7].

\footnotetext{
AMS Mathematics subject classification. Primary 45C05, 45E05, 30C40.

Keywords and phrases. Kerzman-Stein operator, norm, eigenvalues.

Received by the editors on June 7, 2006, and in revised form on September 6, 2006.

Copyright (C)2007 Rocky Mountain Mathematics Consortium
} 
In a later article [6], Kerzman asked a number of questions about this operator, including the following.

Problem [6]. Relate the spectrum of the Kerzman-Stein operator to the geometry of the domain.

In a sense, the spectrum measures the error when the Cauchy kernel is used to approximate the Szegö kernel and may be useful for estimating the rate of convergence of certain solutions to the Riemann map.

In this article we establish a lower estimate for the norm of the Kerzman-Stein operator.

Theorem 1. For a continuously differentiable, multiply connected domain $\Omega \subset \subset \mathbf{C}$, the Kerzman-Stein operator satisfies

$$
\|\mathcal{A}\| \geq \sqrt{\frac{\text { length }(\partial \Omega)}{2 \pi d}-1}
$$

where $d$ is the capacity of $\bar{\Omega}$.

For a disc, it is known that $\mathcal{A}$ is trivial, and in this case there is equality since the capacity is the same as the radius. Related to this, the Kerzman-Stein operator is invariant under Möbius transformations, although the estimate is not. (It is, however, invariant under dilations.) A stronger estimate, then, that also permits $\Omega$ to be unbounded, is as follows.

Theorem 2. For a continuously differentiable, finitely connected domain $\Omega$, the Kerzman-Stein operator satisfies

$$
\|\mathcal{A}\| \geq \sup _{\mu} \sqrt{\frac{\operatorname{length}\left(\partial \Omega^{\prime}\right)}{2 \pi d^{\prime}}-1}
$$

where the supremum is taken over the bounded Möbius images $\Omega^{\prime}=$ $\mu(\Omega)$ of $\Omega$, and $d^{\prime}$ is the capacity of $\overline{\Omega^{\prime}}$.

We will show that these estimates are sharp for an annulus and a strip, and in limiting situations, they give sharp estimates for an ellipse and a wedge. 
Our proof of Theorem 1 mirrors the proof of an estimate that Bergman and Schiffer gave for the first Fredholm eigenvalue [2, page 228]. It uses the equivalence of the Kerzman-Stein eigenvalue problem $f=-i \lambda \mathcal{A} f$ with the problem of finding functions $u, v$ in the Hardy space satisfying

$$
\begin{aligned}
& v(z)=-\frac{\lambda}{2 \pi} \int_{\partial \Omega} \frac{\overline{u(\eta)}}{\eta-z} d s_{\eta}, \\
& u(z)=+\frac{\lambda}{2 \pi} \int_{\partial \Omega} \frac{\overline{v(\eta)}}{\eta-z} d s_{\eta}
\end{aligned}
$$

that was studied by Singh in [13]. The author anticipates that by drawing on other methods developed for the Fredholm eigenvalues, see $[11,12,15]$, and by exploiting similarities between Singh's problem and the Fredholm eigenvalue problem, one might obtain more refined estimates for $\mathcal{A}$.

Theorem 2 follows directly from Theorem 1 . For the Möbius invariance of $\mathcal{A}$, see [5, page 106] or [13, page 380].

The paper is structured as follows. In Section 2, we prove the equivalence of the Kerzman-Stein eigenvalue problem with the problem of Singh. This was proved earlier by Burbea [5], but the proof here illustrates how the result is primarily a result from functional analysis. In Section 3, we prove Theorem 1; we do this in the context of Singh's problem so that the similarity with the Bergman-Schiffer estimate is clearly evident. In Section 4, we evaluate the estimates for examples the author studied previously $[\mathbf{3}, \mathbf{4}]$. In certain cases the estimates are sharp.

For an excellent treatment of complex analysis and its applications that uses the perspective of the Cauchy operator, see Bell's book [1].

2. Equivalence with the eigenvalue problem of Singh. In this section we prove the equivalence of Singh's eigenvalue problem with the problem $f=-i \mathcal{A} \lambda f$. This was proved earlier by Burbea [5]. Our proof, however, illustrates how the equivalence is primarily a result from functional analysis.

The equivalence follows from the following two lemmas. In our application, we will use the Hilbert space $H=L^{2}(\partial \Omega)$, the Hardy 
space $E=L^{2}(\partial \Omega) \cap O(\Omega)$, the Cauchy projector $\mathcal{C}$ defined using the nontangential interior limit of the Cauchy integral, and the involution $\mathcal{J}: H \rightarrow H$ defined by $f \rightarrow \overline{f T}$ where $T=T(w)$ is the unit tangent vector at $w \in \partial \Omega$. Then $\mathcal{C}^{*}=\mathcal{I}-\mathcal{J C} \mathcal{J}$, as proved by Bell $[\mathbf{1}$, page 10]. Moreover, by the Plemelj theorem [1, page 14], $\mathcal{C}=\mathcal{I} / 2+\mathcal{C}_{0}$, so $\mathcal{A} \stackrel{\text { def }}{=} \mathcal{C}_{0}-\mathcal{C}_{0}^{*}=\mathcal{C}-\mathcal{C}^{*}$. We mention that the Plemelj theorem can also be used to show $\mathcal{C}^{*}=\mathcal{I}-\mathcal{J C} \mathcal{J}$ in case $\Omega$ is only continuously differentiable. See Muskhelishvili [9, page 43], for instance.

Lemma 1. Let $\mathcal{C}$ be a densely defined projection from a Hilbert space $H$ to a closed subspace $E$ whose skew-hermitian part $\mathcal{A}=\mathcal{C}-\mathcal{C}^{*}$ is compact. Here $\mathcal{C}^{*}$ is defined formally. Then $\mathcal{C}$ is bounded, and the orthogonal projection to the same subspace $E$ is given by $\mathcal{S}=$ $\mathcal{C}(\mathcal{I}+\mathcal{A})^{-1}$.

Proof. By the spectral theorem, $(\mathcal{I}+\mathcal{A})^{-1}$ exists and is bounded. We first show that $\mathcal{C}$ is also bounded. So let $u$ be in the domain of $\mathcal{C}$. Then,

$$
\begin{aligned}
\|\mathcal{C} u\|^{2} & =(\mathcal{C} u, \mathcal{C} u)=\left(\left(\mathcal{C}^{*}+\mathcal{A}\right) u, \mathcal{C} u\right)=(u,(\mathcal{C}-\mathcal{A}) \mathcal{C} u) \\
& =\left(u, \mathcal{C}^{2} u\right)-(u, \mathcal{A C} u)=(u, \mathcal{C} u)-(u, \mathcal{A C} u) \\
& \leq\|u\|(\|\mathcal{C} u\|+\|\mathcal{A}\|\|\mathcal{C} u\|)=(1+\|\mathcal{A}\|)\|u\|\|\mathcal{C} u\|
\end{aligned}
$$

and $\|\mathcal{C} u\| \leq(1+\|\mathcal{A}\|)\|u\|$. Then $\mathcal{C}$ is bounded and can be extended to all of $H$ by continuity. Moreover, $\mathcal{C}(\mathcal{I}+\mathcal{A})^{-1}$ is well defined.

We show that this operator is self-adjoint, that is, $\mathcal{C}(\mathcal{I}+\mathcal{A})^{-1}=$ $(\mathcal{I}-\mathcal{A})^{-1} \mathcal{C}^{*}$. To do this, we verify that $(\mathcal{I}-\mathcal{A}) \mathcal{C}=\mathcal{C}^{*}(\mathcal{I}+\mathcal{A})$. The lefthand side of this last equation is $\left(\mathcal{I}-\mathcal{C}+\mathcal{C}^{*}\right) \mathcal{C}=\mathcal{C}^{*} \mathcal{C}$, since $\mathcal{C}^{2}=\mathcal{C}$; the righthand side is $\mathcal{C}^{*}\left(\mathcal{I}+\mathcal{C}-\mathcal{C}^{*}\right)=\mathcal{C}^{*} \mathcal{C}$, since $\left(\mathcal{C}^{*}\right)^{2}=\mathcal{C}^{*}$. So $(\mathcal{I}-\mathcal{A}) \mathcal{C}=\mathcal{C}^{*} \mathcal{C}=\mathcal{C}^{*}(\mathcal{I}+\mathcal{A})$, and $\mathcal{C}(\mathcal{I}+\mathcal{A})^{-1}$ is self-adjoint.

Finally, we show that it reproduces the same functions as $\mathcal{C}$. So suppose $\mathcal{C} u=u$. Then $(\mathcal{I}-\mathcal{A}) u=\left(\mathcal{I}-\mathcal{C}+\mathcal{C}^{*}\right) u=\mathcal{C}^{*} u$ and $u=$ $(\mathcal{I}-\mathcal{A})^{-1} \mathcal{C}^{*} u$. By the last paragraph, we then have $u=\mathcal{C}(\mathcal{I}+\mathcal{A})^{-1} u$. So the lemma is proved.

We mention that the relations between the skew projection, its adjoint, and the orthogonal projection on the same subspace have been 
considered previously. See, for instance, Theorem 6.1 and its proof in Spitkovsky [14].

Lemma 2. Suppose further that $\mathcal{J}: H \rightarrow H$ is an anti-linear involution which maps $E \rightarrow E^{\perp}$ and $E^{\perp} \rightarrow E$ with $\mathcal{C}^{*}=\mathcal{I}-\mathcal{J C} \mathcal{J}$. Then the eigenvalue problem $f=-i \lambda \mathcal{A} f$ for $f \in H$ is equivalent to the eigenvalue problem $v=-i \lambda \mathcal{C} \mathcal{J} u, u=+i \lambda \mathcal{C} \mathcal{J} v$ for $u, v \in E$.

Proof. $(\Rightarrow)$. Suppose first that $f=-i \lambda \mathcal{A} f$ for $f \in H$ and $\lambda \in \mathbf{R}$. Define $v=\mathcal{S} f$ and $u=\mathcal{J}(\mathcal{I}-\mathcal{S}) f$. Then both $u, v \in E$, and

$$
v=\mathcal{S}(-i \lambda \mathcal{A} f)=-i \lambda \mathcal{S} \mathcal{A} f=-i \lambda \mathcal{C}(\mathcal{I}-\mathcal{S}) f=-i \lambda \mathcal{C} \mathcal{J} u
$$

The third equality follows from

$\mathcal{S A}=\mathcal{S C}-\mathcal{S C}^{*}=\mathcal{C}-(\mathcal{C S})^{*}=\mathcal{C}-\mathcal{S}^{*}=\mathcal{C}-\mathcal{S}=\mathcal{C}-\mathcal{C S}=\mathcal{C}(\mathcal{I}-\mathcal{S})$

So we have left to check that $u=i \lambda \mathcal{C} \mathcal{J} v$. Notice that

$$
u=\mathcal{J}(\mathcal{I}-\mathcal{S}) f=\mathcal{J}(\mathcal{I}-\mathcal{S})(-i \lambda \mathcal{A} f)=i \lambda \mathcal{J}(\mathcal{I}-\mathcal{S}) \mathcal{A} f
$$

by the anti-linearity of $\mathcal{J}$. Moreover,

$$
(\mathcal{I}-\mathcal{S}) \mathcal{A}=\mathcal{C}-\mathcal{C}^{*}-\mathcal{S C}+\mathcal{S C}{ }^{*}=\mathcal{C}-\mathcal{C}^{*}-\mathcal{C}+(\mathcal{C S})^{*}=-\mathcal{C}^{*}+\mathcal{S},
$$

so that $u=i \lambda \mathcal{J}\left(-\mathcal{C}^{*}+\mathcal{S}\right) f$. Since $\mathcal{C}^{*}=\mathcal{I}-\mathcal{J C} \mathcal{J}$, we next have

$$
\mathcal{J}\left(-\mathcal{C}^{*}+\mathcal{S}\right)=\mathcal{C} \mathcal{J}-\mathcal{J}(\mathcal{I}-\mathcal{S})=\mathcal{C} \mathcal{J}-\mathcal{C} \mathcal{J}(\mathcal{I}-\mathcal{S})=\mathcal{C} \mathcal{J} S
$$

It follows that $u=i \lambda \mathcal{C} \mathcal{J S} f=i \lambda \mathcal{C} \mathcal{J} v$ as needed.

$(\Leftarrow)$. Suppose, then, that $u, v \in E$ satisfy $v=-i \lambda \mathcal{C} \mathcal{J} u$ and $u=+i \lambda \mathcal{C} \mathcal{J} v$. Set $f=v+\mathcal{J} u$. Then,

$f=-i \lambda \mathcal{C} \mathcal{J} u+\mathcal{J}(i \lambda \mathcal{C} \mathcal{J} v)=-i \lambda(\mathcal{C} \mathcal{J} u+\mathcal{J C} \mathcal{J} v)=-i \lambda\left(\mathcal{C} \mathcal{J} u+\left(\mathcal{I}-\mathcal{C}^{*}\right) v\right)$

Meanwhile,

$\mathcal{A} f=\left(\mathcal{C}-\mathcal{C}^{*}\right)(v+\mathcal{J} u)=\mathcal{C} v+\mathcal{C} \mathcal{J} u-\mathcal{C}^{*} v-\mathcal{C}^{*} \mathcal{J} u=\mathcal{C} \mathcal{J} u+\left(\mathcal{I}-\mathcal{C}^{*}\right) v$ 
where $\mathcal{C} v=v$ and $\mathcal{C}^{*} \mathcal{J} u=0$ since $u, v \in E$. It follows that $f=-i \lambda \mathcal{A} f$, and the lemma is proved.

3. Proof of Theorem 1. The proof of Theorem 1 mirrors the proof of an estimate that Bergman and Schiffer gave for the first Fredholm eigenvalue [2, page 228]. It depends on the formulation of the problem that was studied by Singh. We refer to Ransford [10] for the following general properties of the capacity $c=c(K)$.

1. If $K$ is a compact subset of $\mathbf{C}$, then $c(K)=c\left(\partial_{e} K\right)$ where $\partial_{e} K$ indicates the exterior boundary of $K$ [10, page 128].

2. If $K_{1}, K_{2}$ are compact subsets of $\mathbf{C}$ and $D_{1}, D_{2}$ are the unbounded components of $\mathbf{C} \backslash K_{1}$ and $\mathbf{C} \backslash K_{2}$, respectively, and if there is a meromorphic function $f: D_{1} \rightarrow D_{2}$ with $f(z)=z+O(1)$ as $z \rightarrow \infty$, then $c\left(K_{2}\right) \leq c\left(K_{1}\right)$. There is equality if $f$ is conformal from $D_{1}$ onto $D_{2}[\mathbf{1 0}$, page 133].

3. If $K$ is a disc of radius $r$, then $c(K)=r$ [10, page 135].

Proof of Theorem 1. Suppose that $\Omega \subset \subset \mathbf{C}$ is bounded by curves $C_{1}, C_{2}, \ldots, C_{k}$, each of which is continuously differentiable. Let $\Omega^{c}=$ $\mathbf{C} \backslash \bar{\Omega}$ be the domain complement of $\Omega$, and let $\Omega_{j}^{c} \subset \Omega^{c}$ be the component of $\Omega^{c}$ whose boundary is $C_{j}$. Choose $C_{1}$ to be the curve for which $\Omega_{1}^{c}$ is the unbounded component of $\Omega^{c}$. Finally, let $\Omega_{1}=$ $\Omega \cup\left(\cup_{j \neq 1} \overline{\Omega_{j}^{c}}\right)$. This is the region bounded by $C_{1}$ that also contains $\Omega$.

Let $z=f(\zeta)=d\left(\zeta+c_{0}+c_{1} \zeta^{-1}+\cdots\right)$ be the function which maps the domain $|\zeta|>1$ conformally onto $\Omega_{1}^{c}$ with $d>0$. Using the properties mentioned above, we have $c(\bar{\Omega})=c\left(\overline{\Omega_{1}}\right)=d$.

We next pass to the complementary domain $\Omega^{c}$ for which the eigenvalue problems are completely equivalent; in particular, the eigenvalues for $\Omega$ are also the eigenvalues for $\Omega^{c}$. For Singh's problem this is shown on [13, page 380]. Let $K(z, \bar{\xi})$ denote the Szegő kernel for $\Omega^{c}$, and let

$$
\Gamma(z, \bar{\xi})=\frac{1}{4 \pi^{2}} \int_{\partial \Omega^{c}} \frac{d s_{\eta}}{(\eta-z)(\bar{\eta}-\bar{\xi})} .
$$

Then $\Gamma(z, \bar{\xi})-K(z, \bar{\xi})$ is a hermitian, positive semi-definite kernel. See [13, page 377]. In fact, if $\lambda_{j}, u_{j}$, and $v_{j}$, for $j>0$, are solutions of Singh's eigenvalue problem for $\Omega^{c}$, with $0<\lambda_{1} \leq \lambda_{2} \leq \cdots \rightarrow \infty$, then 
there are series expansions

$$
K(z, \bar{\xi})=\sum_{j>0}\left(u_{j}(z) \overline{u_{j}(\xi)}+v_{j}(z) \overline{v_{j}(\xi)}\right),
$$

and

$$
\Gamma(z, \bar{\xi})=\sum_{j>0}\left(1+\frac{1}{\lambda_{j}^{2}}\right)\left(u_{j}(z) \overline{u_{j}(\xi)}+v_{j}(z) \overline{v_{j}(\xi)}\right) .
$$

Furthermore, $0<\lambda_{1} \leq \lambda_{j}$ implies $1 / \lambda_{1}^{2} \geq 1 / \lambda_{j}^{2}$, so that $\Gamma(z, \bar{z}) \leq$ $\left(1+\left(1 / \lambda_{1}^{2}\right)\right) K(z, \bar{z})$.

Next, notice from (1) that as $|z| \rightarrow \infty$,

$$
\Gamma(z, \bar{z})|z|^{2}=\frac{1}{4 \pi^{2}} \int_{\partial \Omega^{c}} \frac{|z|^{2}}{|\eta-z|^{2}} d s_{\eta} \longrightarrow \frac{1}{4 \pi^{2}} \text { length }\left(\partial \Omega^{c}\right) .
$$

To manage the Szegő kernel we first write

$$
K(z, \bar{\xi})=K_{1}(z, \bar{\xi})+\sum_{j \neq 1} K_{j}(z, \bar{\xi})
$$

where $K_{j}(z, \bar{\xi})$ is the Szegö kernel for $\Omega_{j}^{c}$ and is assumed to vanish if either $z, \xi \notin \overline{\Omega_{j}^{c}}$. Then for large $|z|$ we have $K(z, \bar{z})=K_{1}(z, \bar{z})$. Next, since the Szegó kernel for the complement of the unit disc is given by

$$
K_{\Delta^{c}}(\zeta, \bar{w})=\frac{1}{2 \pi} \frac{1}{\zeta \bar{w}-1}
$$

we have, by the transformation formula for the Szegő kernel (see $[\mathbf{1}$, page 44]), that

$$
K_{1}(z, \bar{z})\left|f^{\prime}(\zeta)\right|=\frac{1}{2 \pi} \frac{1}{\zeta \bar{\zeta}-1} .
$$

So for large $|z|, K(z, \bar{z})|z|^{2}=|z|^{2} /\left|f^{\prime}(\zeta)\right| \cdot(2 \pi)^{-1}(\zeta \bar{\zeta}-1)^{-1}$, and this approaches

$$
\frac{d^{2}|\zeta|^{2}}{d} \cdot \frac{1}{2 \pi} \frac{1}{\zeta \bar{\zeta}-1} \approx \frac{d}{2 \pi}
$$

as $|z| \rightarrow \infty$. It follows that

$$
\frac{\Gamma(z, \bar{z})}{K(z, \bar{z})}=\frac{\Gamma(z, \bar{z}) \cdot|z|^{2}}{K(z, \bar{z}) \cdot|z|^{2}} \rightarrow \frac{1}{4 \pi^{2}} \text { length }\left(\partial \Omega^{c}\right) \cdot \frac{2 \pi}{d}=\frac{1}{2 \pi} \frac{\text { length }(\partial \Omega)}{d},
$$


as $|z| \rightarrow \infty$, and

$$
\frac{1}{2 \pi} \frac{\operatorname{length}(\partial \Omega)}{d} \leq 1+\frac{1}{\lambda_{1}^{2}} .
$$

Solving this inequality gives

$$
\|\mathcal{A}\|=\frac{1}{\lambda_{1}} \geq \sqrt{\frac{1}{2 \pi} \frac{\text { length }(\partial \Omega)}{d}-1},
$$

and the theorem is proved.

4. Examples. Previously, the author gave descriptions of the Kerzman-Stein operator for certain model domains $[\mathbf{3}, 4]$. Here we evaluate the estimates of Theorems 1 and 2 for these known cases. The estimates are sharp for an annulus and a strip; they are asymptotically sharp for an ellipse and a wedge.

4.1 Annulus. In [4] it was shown that the spectrum of $\mathcal{A}$ for an annulus $\left\{z \in \mathbf{C}: r<|z|<r^{-1}\right\}, 0<r<1$, consists of the values $\pm i r^{2 n-1}$ for $n=1,2, \ldots$, and therefore $\|\mathcal{A}\|=r$. Meanwhile, the annulus has boundary length $2 \pi\left(r+r^{-1}\right)$ and capacity $r^{-1}$. In this case, the estimate says

$$
\|\mathcal{A}\| \geq \sqrt{\frac{2 \pi\left(r+r^{-1}\right)}{2 \pi \cdot r^{-1}}-1}=\sqrt{r^{2}+1-1}=r,
$$

which is evidently sharp. We mention that Singh also computed the eigenvalues for the annulus in [13, page 392].

4.2 Strip. In $[4]$ it was shown that the spectrum of $\mathcal{A}$ for a strip $\{z \in \mathbf{C}:|\Im z|<1\}$ consists of the continuous interval $[-i,+i]$ on the imaginary axis. In this case, $\|\mathcal{A}\|=1$. Using a Möbius transformation, however, the strip can be mapped to the region bounded between internally tangent circles. For instance, $\mu=2 r /[i(1-r) z+r+1]$ maps the strip to the region between circles $|z-1 / 2|=1 / 2$ and $|z-r / 2|<r / 2$, for $0<r<1$. This region has boundary length $\pi(1+r)$ and capacity $1 / 2$. The estimate then says

$$
\|\mathcal{A}\| \geq \sqrt{\frac{\pi(1+r)}{2 \pi \cdot 1 / 2}-1}=\sqrt{r} .
$$


As $r \rightarrow 1^{-}$the righthand side approaches 1 , so the estimate of Theorem 2 is sharp.

4.3 Ellipse. In $[3]$ it was shown that the spectrum of $\mathcal{A}$ for an ellipse with major and minor axes $2(1+r)$ and $2(1-r)$, respectively, consists of the values $\pm i \beta_{n} r^{2 n-1}+o\left(r^{2 n-1}\right)$ valid asymptotically as $r \downarrow 0$, for certain $0<\beta_{n} \leq 1$ and $n=1,2, \ldots$ In particular, the values that are largest by absolute value are $\pm i r / 2+o(r)$ so that $\|\mathcal{A}\|=r / 2+o(r)$, valid as $r \downarrow 0$. (These are the ellipses with small eccentricity.) Meanwhile, one finds that the ellipse has boundary length $2 \pi\left(1+r^{2} / 4\right)+O\left(r^{3}\right)$ and capacity 1 . In this case, the estimate says

$$
\|\mathcal{A}\| \geq \sqrt{\frac{2 \pi\left(1+r^{2} / 4\right)+O\left(r^{3}\right)}{2 \pi \cdot 1}-1}=\frac{r}{2}+O\left(r^{2}\right) .
$$

Evidently, the estimate of Theorem 1 is asymptotically sharp for $r \downarrow 0$.

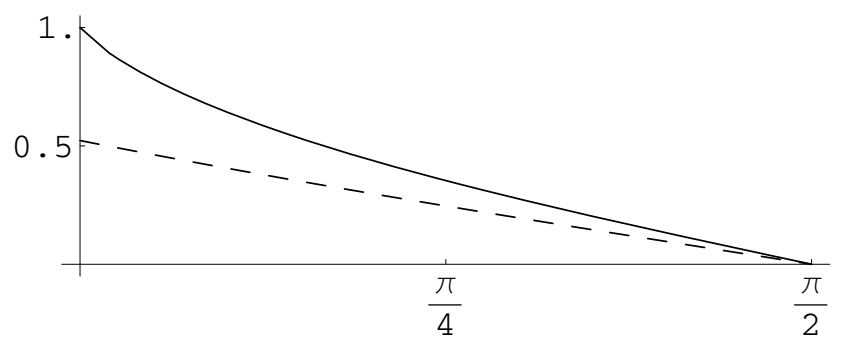

FIGURE 1. Analysis of the estimate for a wedge, $0<\theta<\pi / 2$.

4.4 Wedge. In [4] it was shown that the spectrum of $\mathcal{A}$ for a wedge $\{z \in \mathbf{C}:|\operatorname{Arg} z|<\theta\}, 0<\theta<\pi / 2$, consists of the continuous interval on the imaginary axis given as the range of values of $i \psi(\xi)=i \sinh [\xi(\pi-2 \theta)] / \cosh (\xi \pi), \xi \in \mathbf{R}$. The norm is $\|\mathcal{A}\|=$ $\max _{\xi \in \mathbf{R}} \sinh [\xi(\pi-2 \theta)] / \cosh (\xi \pi)$.

Using a Möbius transformation, however, the wedge can be mapped to a lens with vertices at \pm 1 . One finds that this lens has boundary length $4 \theta \csc \theta$. It also has capacity $\pi /(2(\pi-\theta))$. (For the capacity of the lens, see [10, page 135]; the domain is the lune with $h=2$ and $\beta=\theta=-\alpha$.) In this case the estimate of Theorem 1 says

$$
\|\mathcal{A}\| \geq \sqrt{\frac{4(\pi-\theta) \theta \csc \theta}{\pi^{2}}-1 .}
$$


Figure 1 illustrates the estimate for wedges $0<\theta<\pi / 2$. The actual norm of $\mathcal{A}$ is indicated by the solid graph; the lower bound is indicated by the dotted graph. Evidently the estimate is sharp in the limiting case $\theta \rightarrow \pi / 2$. This corresponds with wedges that approach a half-plane, where $\mathcal{A}$ is trivial.

\section{REFERENCES}

1. Steven R. Bell, The Cauchy transform, potential theory, and conformal mapping, Stud. Adv. Math., CRC Press, Boca Raton, FL, 1992.

2. S. Bergman and M. Schiffer, Kernel functions and conformal mapping, Comp. Math. 8 (1951), 205-249.

3. Michael Bolt, Spectrum of the Kerzman-Stein operator for the ellipse, Integral Equations Operator Theory, 57 (2007), 167-184.

4. - Spectrum of the Kerzman-Stein operator for model domains, Integral Equations Operator Theory 50 (2004), 305-315.

5. Jacob Burbea, The Cauchy and the Szegő kernels on multiply connected regions, Rend. Circ. Mat. Palermo 31 (1982), 105-118.

6. N. Kerzman, Singular integrals in complex analysis, in Harmonic analysis in Euclidean spaces Proc. Sympos. Pure Math. 35, 3-41, Amer. Math. Soc., Providence, 1979.

7. N. Kerzman and E.M. Stein, The Cauchy kernel, the Szegö kernel, and the Riemann mapping function, Math. Ann. 236 (1978), 85-93.

8. Loredana Lanzani, Szegő projection versus potential theory for non-smooth planar domains, Indiana Univ. Math. J. 48 (1999), 537-555.

9. N.I. Muskhelishvili, Singular integral equations, Dover Publications Inc., New York, 1992.

10. Thomas Ransford, Potential theory in the complex plane, London Math. Soc. Student Texts 28, Cambridge University Press, Cambridge, 1995.

11. M. Schiffer, The Fredholm eigen values of plane domains, Pacific J. Math. 7, 1957.

12. M. Schiffer and G. Schober, An extremal problem for the Fredholm eigenvalues, Arch. Rational Mech. Anal. 44 (1971/72), 83-92.

13. Vikramaditya Singh, An integral equation associated with the Szegö kernel function, Proc. London Math. Soc. 10 (1960), 376-394.

14. Ilya Spitkovsky, Once more on algebras generated by two projections, Linear Algebra Appl. 208/209 (1994), 377-395.

15. George Springer, Fredholm eigenvalues and quasiconformal mapping, Acta Math. 111 (1964), 121-142. 
Department of Mathematics and Statistics, 1740 Knollcrest Circle SE, Calvin College, Grand Rapids, Michigan 49546-4403

Email address: mbolt@calvin.edu 